\title{
Some Properties of Multi-Qubit States in Non-Inertial Frames
}

\author{
Alaa Sagheer $\Psi^{\ddagger \ddagger}$ and Hala Hamdoun ${ }^{\ddagger}$ \\ ${ }^{\dagger}$ Department of Mathematics \\ ${ }^{\ddagger}$ Center for Artificial Intelligence and RObotics (CAIRO) \\ Faculty of Science, Aswan University, Aswan, Egypt \\ Email: \{alaa, hala\}@cairo-svu.edu.eg
}

\begin{abstract}
In this paper, some properties of multi-qubit states traveling in non-inertial frames are investigated, where we assume that all particles are accelerated. These properties are including fidelities, capacities and entanglement of the accelerated channels for three different states, namely, Greeberger-Horne-Zeilinger (GHZ) state, GHZ-like state and $\mathrm{W}$-state. It is shown here that all these properties are decreased as the accelerations of the moving particles are increased. The obtained results show that the GHZ-state is the most robust one comparing to the others, where the degradation rate is less than that for the other states particularly in the second Rindler region. Also, it is shown here that the entangled property doesn't change in the accelerated frames. Additionally, the paper shows that the degree of entanglement decreases as the accelerations of the particles increase in the first Rindler region. However in the second region, where all subsystems are disconnected at zero acceleration, entangled channels are generated as the acceleration increases.
\end{abstract}

Keywords: Multi-qubit states, Non-inertial frame, Fidelity, Capacity, Entanglement, GHZ state, GHZ-like state, W-state.

\section{Introduction}

Recently, quantum information theory in the relativistic framework has attracted considerable attention. It seems to be mainly due to the fact that many modern experiments on quantum information processing involve the use of photons and/or electrons, where the relativistic effect is not negligible [1]. The dynamics of entanglement and its applications for traveling systems in non-inertial frames have been investigated by many authors. For example, Alsing et al. (2003) used the accelerated channel to perform quantum teleportation [2]. Landulf et al. (2009) discussed the phenomena of entanglement sudden death and information lose for a two qubits system in the non-inertial frames [3]. Khan et al. (2011) investigated the relativistic quantum game in the non-inertial frames, where the impact of the Unruh effect on the non-zero sum games was discussed [4]. Metwally (2012) discussed the usefulness of the travelling channels in the non-inertial frames to perform effective quantum teleportation of accelerated and non accelerated information [5, 6].

Futhermore, the dynamics of tripartite qubits in the non-inertial frames have been discussed from different point of views. For example, Hwang et al. (2001) examined the tripartite entanglement when one of the three parties moves with a uniform acceleration with respect to the other parties [7]. Esfahani et al. (2012) discussed the dynamics of entanglement for three spin $\frac{1}{2}$ massive particles by Gaussian momentum distribution [8].

In this paper, we investigate the behavior of the tripartite entanglement in the noninertial frames through GHZ state, GHZ-like state and W-state,where these states could be used to perform many tasks of quantum information. Also, we investigate the fidelity property and the channel capacity property of these three states in the accelerated frames.

${ }^{*}$ Corresponding author 
The outline of this paper is as follows: An analytical solution for the proposed model for the different three states is introduced in Sec.2. The behavior of the fidelities and the capacities of the accelerated channels are described in Sec.3. Quantifying the degree of entanglement of the accelerated channels is provided in Sec.4. Finally, Sec.5 concludes this paper.

\section{The Proposed Model}

In this section we investigate the behavior of the three different types of multi-qubit states, GHZ, GHZ-like and W-states, in non-inertial frames. These tripartite states have been classified by Dür et. al [9], such that they can't be obtained from each other by using local operation and classical communication. These states have been widely as quantum channels to perform different quantum information and computations tasks. For example, Karlesson et al. [10] showed that GHZ state can be utilized to establish teleportation. Due to the new technology, the GHZ state is used as quantum channel to teleport quantum correlation be means of what is called entangement swapping [11].

Assume that we have three users Alice, Bob and Chiral share one of a pure state in the form:

$$
\begin{aligned}
\left|\psi_{w}\right\rangle & =\frac{1}{\sqrt{3}}(|001\rangle+|010\rangle+|100\rangle), \\
\left|\psi_{G}\right\rangle & =\frac{1}{\sqrt{2}}(|000\rangle+|111\rangle), \\
\left|\psi_{G L}\right\rangle & =\frac{1}{2}(|001\rangle+|010\rangle+|100\rangle+|111\rangle) .
\end{aligned}
$$

where $\left|\psi_{w}\right\rangle,\left|\psi_{G}\right\rangle$ and $\left|\psi_{G L}\right\rangle$ represent the W, GHZ and GHZ-like states respectively. It is assumed that these states represent three qubits state of fermions particle of mass $m$ moves in the Mikowski space. Let us consider that these states represent a solution for Dirac equation:

$$
i \gamma^{\kappa}\left(\partial_{\kappa}-\Gamma_{\kappa}\right) \psi+m \psi=0
$$

where $\gamma^{\kappa}$ represents the Dirac matrices, $\Gamma_{\kappa}$ are spinorial affine connections and $\psi$ is a spinor wave functions [12, 13]. The transformations from Minkowski space to Rindler space are given by:

$$
\left|0_{k}\right\rangle_{M}=\cos r\left|0_{k}^{+}\right\rangle_{I}\left|0_{k}^{-}\right\rangle_{I I}+e^{-i \phi} \sin r\left|1_{k}^{+}\right\rangle_{I}\left|1_{k}^{-}\right\rangle_{I I}, \quad\left|1_{k}\right\rangle_{M}=\left|1_{k}^{+}\right\rangle_{I}\left|0_{k}^{-}\right\rangle_{I I},
$$

where $k=a, b$ and $c$. These transformations divide Rindler space into two regions $I$ and $I I$ for fermions and anti-fermions states respectively, (for more details see [14, 5]).

It is known that, the density operator for each state in Eq.(11) is obtained as:

$$
\rho=\sum_{j} p_{j}\left|\psi_{j}\right\rangle\left\langle\psi_{j}\right|
$$

where $p_{j}$ is the probability for the qubit to be in state $\left|\psi_{j}\right\rangle$.

\subsection{W-state:}

The state vector of $\mathrm{W}$-state is given by $\psi_{w}$ from Eq.(1). Then by using the transformation in Eq.(3), we get the W-state vector in both of Rindler regions $I$ and $I I$. By calculating 
the density operator as in Eq.(41) for the W-state and trace out the anti-fermions particles in the second region $I I$, we get the accelerated channel of the initial $\mathrm{W}$ - state, $\rho_{w}$ in the first region $I$ as:

$$
\begin{aligned}
\rho_{W}^{(I)}= & \frac{1}{3}\left\{| 1 0 0 \rangle \left(| C _ { 2 } | ^ { 2 } | C _ { 3 } | ^ { 2 } \left\langle\left.100\left|+C_{2} C_{1}^{*}\right| C_{3}\right|^{2}\left\langle\left. 010\left|+C_{3} C_{1}^{*}\right| C_{2}\right|^{2}\langle 001|\right)\right.\right.\right. \\
& +|010\rangle\left(C _ { 1 } C _ { 2 } ^ { * } | C _ { 3 } | ^ { 2 } \left\langle\left.100|+| C_{1}\right|^{2}\left|C_{3}\right|^{2}\left\langle\left. 010\left|+C_{3} C_{2}^{*}\right| C_{1}\right|^{2}\langle 001|\right)\right.\right. \\
& +|001\rangle\left(C _ { 1 } C _ { 3 } ^ { * } | C _ { 2 } | ^ { 2 } \left\langle\left.100\left|+C_{2} C_{3}^{*}\right| C_{1}\right|^{2}\left\langle\left. 010|+| C_{1}\right|^{2}\left|C_{2}\right|^{2}\langle 001|\right)\right.\right. \\
& +|101\rangle\left(\{ | S _ { 1 } | ^ { 2 } | C _ { 2 } | ^ { 2 } + | C _ { 2 } | ^ { 2 } | S _ { 3 } | ^ { 2 } \} \left\langle\left.101\left|+C_{2} C_{1}^{*}\right| S_{3}\right|^{2}\left\langle\left. 011\left|+C_{2} C_{3}^{*}\right| S_{1}\right|^{2}\langle 110|\right)\right.\right. \\
& +|110\rangle\left(\{ | S _ { 2 } | ^ { 2 } | C _ { 3 } | ^ { 2 } + | S _ { 1 } | ^ { 2 } | C _ { 3 } | ^ { 2 } \} \left\langle\left.110\left|+C_{3} C_{1}^{*}\right| S_{2}\right|^{2}\left\langle\left. 011\left|+C_{3} C_{2}^{*}\right| S_{1}\right|^{2}\langle 101|\right)\right.\right. \\
& +|011\rangle\left(\{ | C _ { 1 } | ^ { 2 } | S _ { 3 } | ^ { 2 } + | C _ { 1 } | ^ { 2 } | S _ { 1 } | ^ { 2 } \} \left\langle\left.011\left|+C_{1} C_{2}^{*}\right| S_{3}\right|^{2}\left\langle\left. 101\left|+C_{1} C_{3}^{*}\right| S_{2}\right|^{2}\langle 110|\right)\right.\right. \\
& \left.+|111\rangle\left(\left|S_{2}\right|^{2}\left|S_{3}\right|^{2}+\left|S_{1}\right|^{2}\left|S_{3}\right|^{2}+\left|S_{1}\right|^{2}\left|S_{2}\right|^{2}\right)\langle 111|\right\},
\end{aligned}
$$

where $C_{i}=\cos r_{j}, S_{i}=\sin r_{j}, i=1,2,3, j=a, b, c$ and $r_{j}$ are the accelerations of the accelerated particles. Similarly if we trace the fermions particles in the first region, $I$, we obtain the state of anti-fermions in the second region $I I$ as:

$$
\begin{aligned}
\rho_{W}^{(I I)}= & \frac{1}{3}\left\{|000\rangle\left(\left|C_{2}\right|^{2}\left|C_{3}\right|^{2}+\left|C_{1}\right|^{2}\left|C_{3}\right|^{2}+\left|C_{1}\right|^{2}\left|C_{2}\right|^{2}\right)\langle 000|\right) \\
& +|010\rangle\left(\{ | S _ { 2 } | ^ { 2 } | C _ { 3 } | ^ { 2 } + | S _ { 2 } | ^ { 2 } | C _ { 1 } | ^ { 2 } \} \left\langle\left.010\left|+S_{2} S_{1}^{*}\right| C_{3}\right|^{2}\left\langle\left. 100\left|+S_{2} S_{3}^{*}\right| C_{1}\right|^{2}\langle 001|\right)\right.\right. \\
& +|001\rangle\left(\{ | C _ { 1 } | ^ { 2 } | S _ { 3 } | ^ { 2 } + | C _ { 2 } | ^ { 2 } | S _ { 3 } | ^ { 2 } \} \left\langle\left.001\left|+S_{3} S_{1}^{*}\right| C_{2}\right|^{2}\left\langle\left. 100\left|+S_{3} S_{2}^{*}\right| C_{1}\right|^{2}\langle 010|\right)\right.\right. \\
& +|101\rangle\left(| S _ { 1 } | ^ { 2 } | S _ { 3 } | ^ { 2 } \left\langle\left.101\left|+S_{1} S_{2}^{*}\right| S_{3}\right|^{2}\left\langle\left. 011\left|+S_{3} S_{2}^{*}\right| S_{1}\right|^{2}\langle 110|\right)\right.\right. \\
& +|100\rangle\left(\{ | S _ { 1 } | ^ { 2 } | C _ { 3 } | ^ { 2 } + | S _ { 1 } | ^ { 2 } | C _ { 2 } | ^ { 2 } \} \left\langle\left.100\left|+S_{1} S_{2}^{*}\right| C_{3}\right|^{2}\left\langle\left. 010\left|+S_{1} S_{3}^{*}\right| C_{2}\right|^{2}\langle 001|\right)\right.\right. \\
& +|110\rangle\left(| S _ { 1 } | ^ { 2 } | S _ { 2 } | ^ { 2 } \left\langle\left.110\left|+S_{1} S_{3}^{*}\right| S_{2}\right|^{2}\left\langle\left. 011\left|+S_{2} S_{3}^{*}\right| S_{1}\right|^{2}\langle 101|\right)\right.\right. \\
& +|011\rangle\left(\left|S_{2}\right|^{2}\left|S_{3}\right|^{2}\left\langle\left. 011\left|+S_{2} S_{1}^{*}\right| S_{3}\right|^{2}\left\langle\left. 101\left|+S_{3} S_{1}^{*}\right| S_{2}\right|^{2}\langle 110|\right)\right\}\right.
\end{aligned}
$$

\subsection{GHZ-state:}

In the GHZ-state, we assume three users share a three qubits state of GHZ type. In the Rindler space and tracing out the states in the second regions, one gets the behavior of $\rho_{G}$ in the first region as:

$$
\begin{aligned}
\rho_{G}^{(I)=} & \frac{1}{2}\left\{\left|c_{3}\right|^{2}\left\{\left|c_{1}\right|^{2}\left(\left|c_{2}\right|^{2}|000\rangle\left\langle\left. 000|+| s_{2}\right|^{2} \mid 010\right\rangle\langle 010|\right)+\left|s_{1}\right|^{2}\left(\left|c_{2}\right|^{2}|100\rangle\left\langle\left. 100|+| s_{2}\right|^{2} \mid 110\right\rangle\langle 110|\right)\right\}\right. \\
& +\left|s_{3}\right|^{2}\left\{\left|c_{1}\right|^{2}\left(\left|c_{2}\right|^{2}|001\rangle\left\langle\left. 001|+| s_{2}\right|^{2} \mid 011\right\rangle\langle 011|\right)+\left|s_{1}\right|^{2}\left(\left|c_{2}\right|^{2}|101\rangle\left\langle\left. 101|+| s_{2}\right|^{2} \mid 111\right\rangle\langle 111|\right)\right\} \\
& \left.+c_{1} c_{2} c_{3}(|000\rangle\langle 111|+| 111\rangle\langle 000|)+|111\rangle\langle 111|\right\} .
\end{aligned}
$$

To find the density operator $\rho_{G}$ in the second region $I I$, we trace out all the modes in the first region $I$. After performing this procedure, one gets, the density operator $\rho_{G}^{(I I)}$ which is the same as $\rho_{G}^{(I)}$ except the last three terms change into $s_{1} s_{2} s_{3}(|000\rangle\langle 111|+$ $|111\rangle\langle 000|)+|000\rangle\langle 000|$. 


\subsection{GHZ-like state:}

The GHZ-like state represents another version of GHZ state, where it can be constructed either from an EPR pair and a single photon or from GHZ state [16]. In the non-inertial frames this state is transformed into $\rho_{G L}$ in the first region $I$ as:

$$
\begin{aligned}
\rho_{G L}^{(I)}= & \frac{1}{4}\left\{|001\rangle\left\{c_{1}^{2} c_{2}^{2}\langle 001|+c_{1} c_{3} c_{2}^{2}\langle 100|+c_{2} c_{3} c_{1}^{2}\langle 010|+c_{1} c_{2} c_{3}\langle 111|\right\}\right. \\
& +|010\rangle\left\{c_{1}^{2} c_{3}^{2}\langle 010|+c_{3} c_{2} c_{1}^{2}\langle 001|+c_{3}^{2} c_{1} c_{2}\langle 100|+c_{1} c_{3}\langle 111|\right\} \\
& +|100\rangle\left\{c_{2}^{2} c_{3}^{2}\langle 100|+c_{3} c_{1} c_{2}^{2}\langle 001|+c_{3}^{2} c_{2} c_{1}\langle 010|+c_{2} c_{3}\langle 111|\right\} \\
& +|101\rangle\left\{\left(s_{1}^{2} c_{2}^{2}+c_{2}^{2} s_{3}^{2}\right)\langle 101|+c_{2} c_{3} s_{1}^{2}\langle 110|+s_{3}^{2} c_{2} c_{1}\langle 011|\right\} \\
& +|110\rangle\left\{\left(s_{2}^{2} c_{3}^{2}+s_{1}^{2} c_{3}^{2}\right)\langle 110|+c_{3} c_{1} s_{2}^{2}\langle 011|+c_{3} c_{2} s_{1}^{2}\langle 101|\right\} \\
& +|011\rangle\left\{\left(c_{1}^{2} s_{2}^{2}+c_{1}^{2} s_{3}^{2}\right)\langle 011|+s_{3}^{2} c_{1} c_{2}\langle 101|+c_{1} c_{3} s_{2}^{2}\langle 110|\right\} \\
& \left.+|111\rangle\left\{\left(s_{1}^{2} s_{2}^{2}+s_{1}^{2} s_{3}^{2}+s_{2}^{2} s_{3}^{2}+1\right)\langle 111|+c_{1} c_{3}\langle 010|+c_{1} c_{2}\langle 001|+c_{2} c_{3}\langle 100|\right\}\right\}
\end{aligned}
$$

On the other hand, the behavior of $\rho_{G L}$ in the second region is described by:

$$
\begin{aligned}
\rho_{G L}^{(I I)}= & \frac{1}{4}\left\{|000\rangle\left(c_{1}^{2} c_{2}^{2}+c_{1}^{2} c_{3}^{2}+c_{2}^{2} c_{3}^{2}+1\right)\langle 000|+s_{1} s_{3}\langle 101|+s_{1} s_{2}\langle 110|+s_{2} s_{3}\langle 011|\right. \\
& +|001\rangle\left\{\left(c_{2}^{2} s_{3}^{2}+c_{1}^{2} s s_{3}^{2}\right)\langle 001|+s_{1} s_{3} c_{2}^{2}\langle 100|+s_{3} s_{2} c_{1}^{2}\langle 010|\right\} \\
& +|010\rangle\left\{\left(s_{2}^{2} c_{3}^{2}+c_{1}^{2} s_{2}^{2}\right)\langle 010|+s_{2} s_{3} c_{1}^{2}\langle 001|+s_{2} s_{1} c_{3}^{2}\langle 100|\right\} \\
& +|100\rangle\left\{\left(s_{1}^{2} c_{2}^{2}+s_{1}^{2} c_{3}^{2}\right)\langle 100|+s_{1} s_{2} c_{3}^{2}\langle 010|+s_{1} s_{3} c_{2}^{2}\langle 001|\right\} \\
& +|101\rangle\left\{s_{1}^{2} s_{3}^{2}\langle 101|+s_{1} s_{3}\langle 000|+s_{1} s_{2} s_{3}^{2}\langle 011|+s_{3} s_{2} s_{1}^{2}\langle 110|\right\} \\
& +|110\rangle\left\{s_{1}^{2} s_{2}^{2}\langle 110|+s_{1} s_{2}\langle 000|+s_{1} s_{3} s_{2}^{2}\langle 011|+s_{2} s_{3} s_{1}^{2}\langle 101|\right\} \\
& \left.+|011\rangle\left\{s_{2}^{2} s_{3}^{2}\langle 011|+s_{2} s_{3}\langle 000|+s_{3}^{2} s_{2} s_{1}\langle 101|+s_{1} s_{3} s_{2}^{2}\langle 110|\right\}\right\}
\end{aligned}
$$

Now, we have obtained the density operators of the accelerated tripartite channels in the non inertial frames. Next section is devoted to investigate some properties of these channels as their fidelities and their capacities in both regions.

\section{Fidelities and Capacities of the Accelerated Chan- nels}

Here, we investigate the behavior of the fidelities and the capacities of the acceleration channels in both Rindler's regions.

It is well known that the fidelity measures the closeness of the accelerated channel in a

specific region to its initial state. Mathematically, the fidelity $\mathcal{F}=\operatorname{tr}\left\{\rho_{\text {initial }} \rho_{\text {final }}\right\}$, where $\rho_{\text {initial }}$ is given by the GHZ, GHZ-like state or W-state and $\rho_{\text {final }}$ is given by the corresponding form of these states in the regions $I$ or $I I$. In this context, the fidelities in the first region are given by:

$$
\begin{aligned}
\mathcal{F}_{W}^{(I)}= & \frac{1}{9}\left\{\left(c_{1}^{2} c_{2}^{2}+c_{1}^{2} c_{3}^{2}+c_{2}^{2} c_{3}^{2}+2 c_{1} c_{2} c_{3}^{2}+2 c_{1} c_{3} c_{2}^{2}+2 c_{3} c_{2} c_{1}^{2}\right\}\right. \\
\mathcal{F}_{G}^{(I)}= & \frac{1}{4}\left\{1+c_{1}^{2} c_{2}^{2} c_{3}^{2}+2 c_{1} c_{2} c_{3}\right\} \\
\mathcal{F}_{G L}^{(I)}= & \frac{1}{16}\left\{1+c_{1}^{2}\left(c_{2}^{2}+c_{3}^{2}\right)+c_{2}^{2} c_{3}^{2}+2 c_{1}\left(c_{2}+c_{3}\right)+2 c_{2} c_{3}+2 c_{1} c_{2} c_{3}^{2}\right. \\
& \left.+2 c_{1} c_{3} c_{2}^{2}+2 c_{2} c_{3} c_{1}^{2}+s_{1}^{2}\left(s_{2}^{2}+s_{3}^{2}\right)+s_{2}^{2} s_{3}^{2}\right\}
\end{aligned}
$$



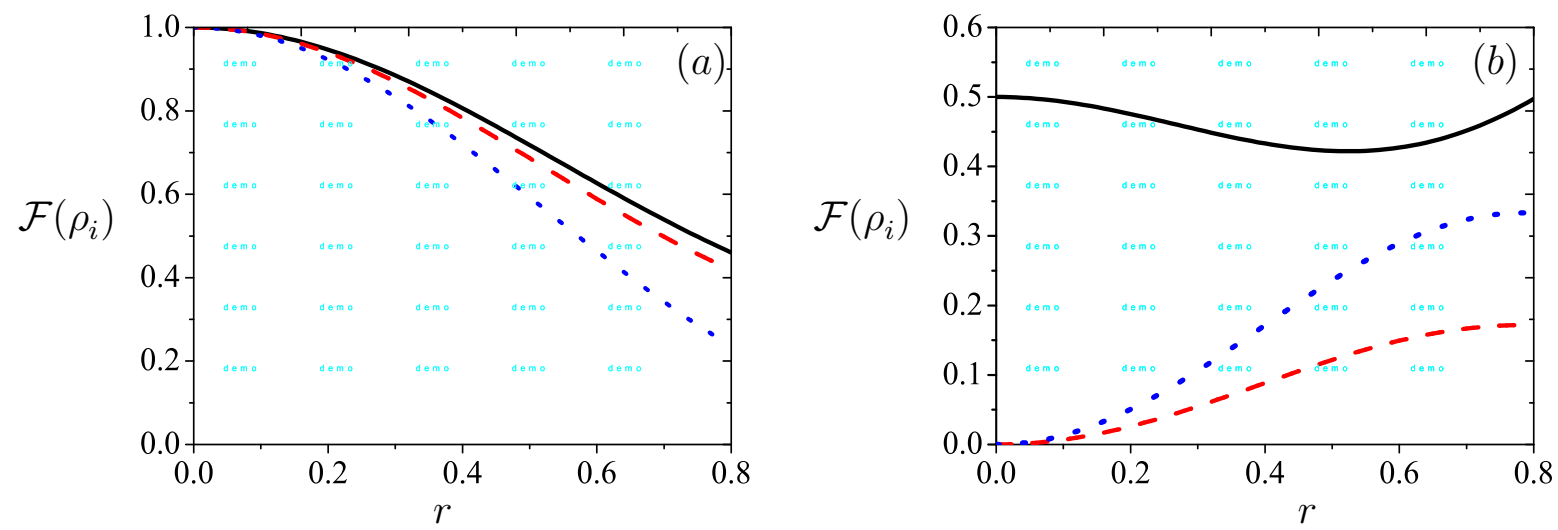

Figure 1: The fidelities of the accelerated states in the first region $I$. The solid, dash and dot curves represent $\mathcal{F}\left(\rho_{G}^{(i)}\right), \mathcal{F}\left(\rho_{G L}^{(i)}\right)$ and $\mathcal{F}\left(\rho_{W}^{(i)}\right)$ respectively, $i=I$ and $I I$.

In the first region, while in the second region $I I$, these fidelities are defined as:

$$
\begin{aligned}
\mathcal{F}_{W}^{(I I)} & =\frac{1}{9}\left\{s_{1}^{2}\left(c_{2}^{2}+c_{3}^{2}\right)+s_{2}^{2}\left(c_{1}^{2}+c_{3}^{2}\right)+s_{3}^{2}\left(c_{1}^{2}+c_{2}^{2}\right)+2 s_{1} s_{3} c_{2}^{2}+2 s_{2} s_{3} c_{1}^{2}+2 s_{1} s_{3} c_{2}^{2}\right\} \\
\mathcal{F}_{G}^{(I I)} & =\frac{1}{4}\left\{\left(1+c_{1}^{2} c_{2}^{2} c_{3}^{2}+s_{1}^{2} s_{2}^{2} s_{3}^{2}+2 s_{1} s_{2} s_{3}\right\},\right. \\
\mathcal{F}_{G L}^{(I I)} & =\frac{1}{16}\left\{s_{1}^{2}\left(c_{2}^{2}+c_{3}^{2}\right)+s_{2}^{2}\left(c_{1}^{2}+c_{3}^{2}\right)+s_{3}^{2}\left(c_{1}^{2}+c_{2}^{2}\right)+2 s_{2} s_{3} c_{1}^{2}+2 s_{1} s_{3} c_{2}^{2}+2 s_{1} s_{2} c_{3}^{2}\right\} .
\end{aligned}
$$

Fig. 1 shows the behavior of the fidelities $\mathcal{F}$ for the accelerated channels in Rindler regions. Fig.1a, describes the behavior of the three tripartite channels in the first region $I$. Generally, it is clear that the fidelities decrease as the accelerations of the moving particles are increased. The minimum values of these fidelities are approached as $r \rightarrow \infty$. These minimum values depend on the type of the accelerated channel. It is easy to notice that the lower bound of the fidelity is bigger for the W-state and smaller for the GHZ and GHZ like state. However the GHZ state has a bigger fidelity than that shown for the GHZ-like state.

The fidelities of the travelling tripartite sates in the second region $I I$, which are descibed by (11) are described in Fig.1b. For the normal GHZ state the initial fidelity $\mathcal{F}_{G}^{(I I)}=0.5$ at $r_{i}=0, i=a, b$ and $c$. However, as the acceleration increases there will be a small change in its behavior. On the other hand, for the GHZ-like state and the $\mathrm{W}$-state, the fidelities are zero for zero accelerations. As the accelerations increase, the fidelities $\mathcal{F}_{G L}^{(I I)}$ and $\mathcal{F}_{W}^{(I I)}$ increase to reach their maximum value as the accelerations tend to $\infty$. The maximum value $\mathcal{F}_{G L}^{(I I)}$ is bigger than that depicted for $\mathcal{F}_{W}^{(I I)}$

All quantum information tasks such as quantum computation and coding information, depend on the capacity of the used quantum channel. Therefore, it is important to evaluate the transmission rate of information from a sender to a receiver. For bipartite state $\rho_{a b}$ the capacity is given by [15]:

$$
\mathcal{C}_{p}=\log _{a} D+\mathcal{S}\left(\rho_{b}\right)-\mathcal{S}\left(\rho_{a b}\right),
$$

where $\rho_{b}=\operatorname{tr}_{a}\left\{\rho_{a b}\right\}, D=2$ is the dimension of $\rho_{a}$ and $\mathcal{S}($.$) is the von Numann entropy.$ For tripartite particles we introduce the average capacity between each two particles as a 

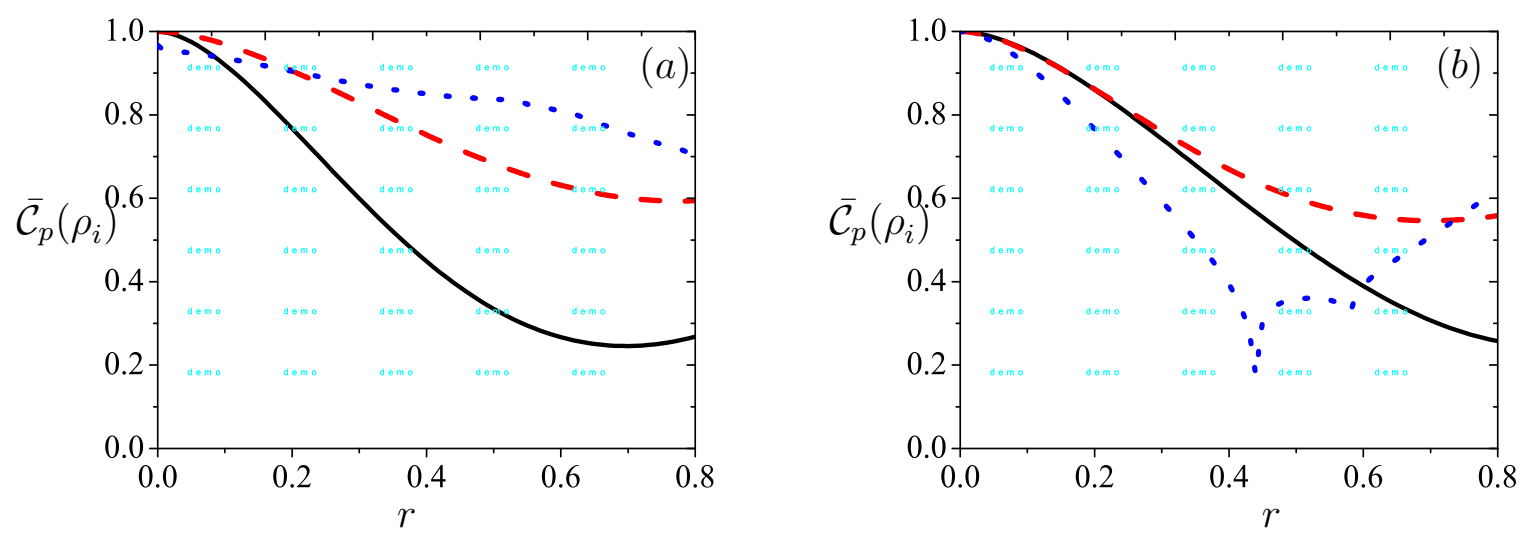

Figure 2: The average capacities $\overline{\mathcal{C}}_{p}(\rho)$ of the accelerated channels in the first Rindler Region (a) $I$ and the second region (b) $I I$. The solid, dash and dot curves represent $\overline{\mathcal{C}_{p}}\left(\rho_{G}^{(i)}\right), \overline{\mathcal{C}}_{p}\left(\rho_{G L}^{(i)}\right)$ and $\overline{\mathcal{C}}_{p}\left(\rho_{W}^{(i)}\right)$ respectively, $i=I$ and $I I$.

measure of the capacity of the accelerated channels. Mathematically, the average capacity is defined as

$$
\overline{\mathcal{C}}_{p}\left(\rho_{a b c}\right)=\frac{1}{3}\left(\mathcal{C}\left(\rho_{a b}\right)+\mathcal{C}\left(\rho_{a b}\right)+\mathcal{C}\left(\rho_{a c}\right)\right)
$$

Fig.2 shows the behavior of the average capacities of the accelerated channel. Fig.2a describes the dynamics of the average capacities in the first Rindler region $I, \overline{\mathcal{C}}_{p}\left(\rho_{i}^{(I)}\right)$, where $i=G, G L$ or $W$ states. As a general behavior the capacities decrease as the accelerations of the particle increase. This means that, the possibility of coding information decreases as the acceleration increases. The diminishing rate is bigger for the normal GHZ state and it is smaller for the $\mathrm{W}$-state. However in the second region a similar behavior is depicted in Fig.2b, but the diminishing rate is smaller than that displayed in Fig.2a.

\section{Entanglement of the Accelerated Channel}

We proceed now for the most important property that we investigate in this paper. We quantify the degree of entanglement of the traveling states in the Rindler regions. To pick up the entanglement features of the bipartite, negativity $\mathcal{N}\left(\rho_{a b c}\right)$ can be adopted as a measure of the degree of entanglement [17, 18, 19]. This measure is defined as

$$
\mathcal{N}\left(\rho_{a b c}\right)=2 \max \left\{0, \sum_{i}\left|\lambda_{i}\right|\right\},
$$

where $\lambda_{i}$ are the negative values of the partial transpose of the density operator $\mathcal{N}\left(\rho_{a b c}\right)$.

The entanglement behavior in the first region $I$ of the three tripartite states is described in Fig.3a, where it is assumed that the three particles are equally accelerated. At zero accelerations i.e. $r_{a}=r_{2}=r_{3}=0$, the fidelity of each state is maximum in the first region $I$, where $\mathcal{N}_{G}^{(I)}=\mathcal{N}_{G L}^{(I)}=1$, while for the W-state $\mathcal{N}_{W}^{(I)} \simeq 0.66$. As the acceleration increases, the entanglement smoothly approachs its minimum values at $r_{i} \rightarrow \infty, i=1,2,3$. It is clear that the degree of entanglement of GHZ-like state is smaller than that shown for the normal GHZ state. However the minimum value of $\mathcal{N}_{\rho_{G L}^{(I)}}$ is smaller than that depicted for $\mathcal{N}_{\rho_{G}}^{(I)}$. The behavior of the W-state is similar than those shown for GHZ family and the $\mathcal{N}\left(\rho_{W}\right)$ is 

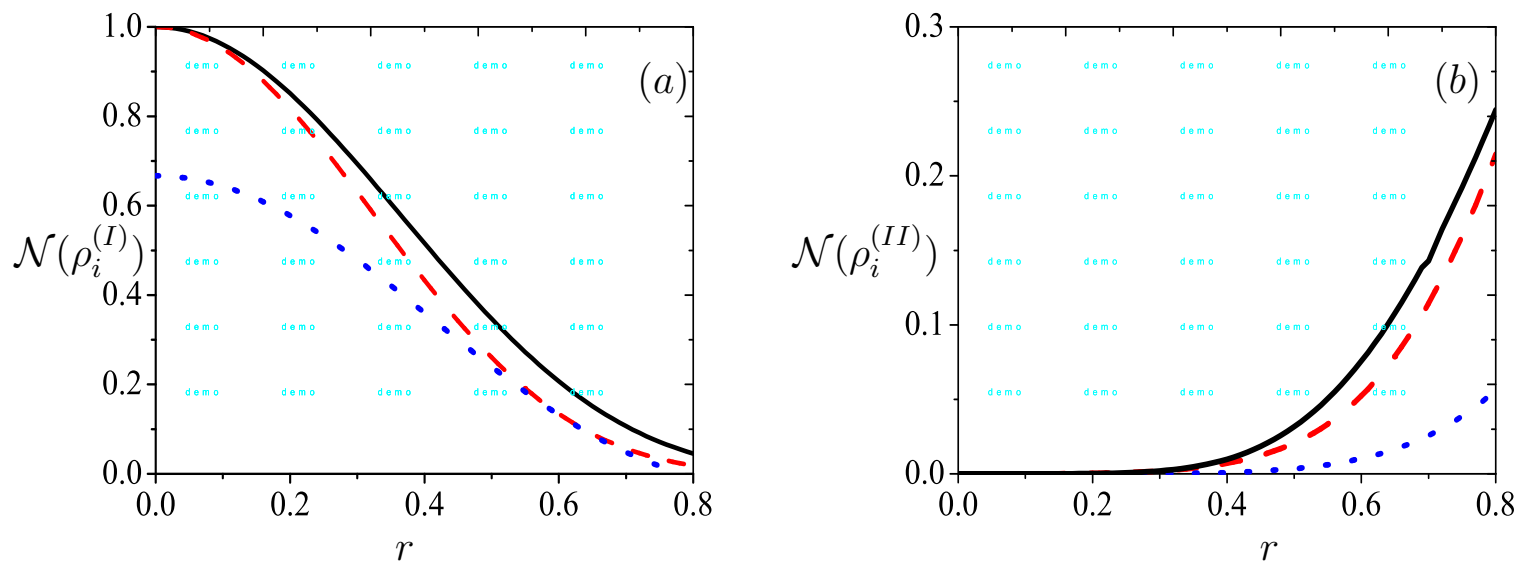

Figure 3: The entanglement of the accelerated channels in the Rindler region(a) $I$ and (b) $I I$. The solid, dash and dot curves represent $\mathcal{N}\left(\rho_{G}^{(i)}\right), \mathcal{N}\left(\rho_{G L}^{(i)}\right)$ and $\mathcal{N}\left(\rho_{W}^{(i)}\right)$ respectively, $i=I$ and $I I$.

completely vanishes as $r_{i} \rightarrow \infty$.

Fig.3b, describes the entanglement of the accelerated channels in Fig.3b, describes the entanglement of the accelerated channels in the second region $I I$. It is clear that at zero acceleration, all the accelerated channels are separable. As the accelerations $r_{i}$ increase, the accelerated channels turns into entangled channels. The degree of entanglement approaches its maximum upper bounds as $r_{i} \rightarrow \infty$

\section{Conclusion}

In this paper, we investigated some properties of GHZ, GHZ-like state and W-state in the non inertial frames, where it is assumed that all the particles are equally accelerated. Namely, we investigated the fidelities, capacities and entanglement. These phenomena have been investigated in both Rindler regions. Analytical expressions have been introduced for these quantities for the different accelerated channels.

It is shown here that, the fidelities of the channels in the first Rindler region decrease as the accelerations of the moving particles increase. However the behavior of the fidelities in the second region increase for the GHZ-like state and the W-state, where the fidelities is completely vanish for small values of the accelerations. The upper limit of the fidelity of the GHZ-like state is bigger than that depicted for the W-state. On the other hand, for the normal GHZ state the fidelity is fluctuated around 0.5. This asserts that the normal GHZ state is more robust in the second region.

The capacities of the accelerated channels are quantified as the average capacities over all the bipartite subsystems. It is shown that the capacities decrease as the accelerations increase. However For the normal GHZ state the degradation is bigger than those depicted for the GHZ-like state and W-state. However the degradation rate in the first Rindler region is bigger than that shown in the second Rindler region. Also, for the normal GHZ state the degradation is the biggest one, while it is the smallest for the W-state. This confirmd that, it is possible to code information in the case of W-state better than that for the case of GHZ and GHZ like states.

Quantifying the degree of entanglement of the accelerated channel in the different Rindler 
region is quantified by means of negativity. In the first rindler's region, the entanglement of the generated entangled channel decreases as the acceleration of the moving particle increases. However, the decay rate of entanglement for GHZ is smaller than that depicted for GHZ-like state. In the second region, there are entangled channels are generated for faster accelerations. The upper limit of the degree of entanglement is the biggest for the GHZ state, while for the W-state it is the smallest.

In conclusion: the entangled properties of the travelling channels don't change in the non inertial frames. Although the W-state has less degree of entanglement, we can coded more information than through the GHZ and the GHZ-like states. The GHZ state is more robust than GHZ-like state.

Acknowledgment: We would like to thank Prof. Abdelmageed Aly and Dr. Saleh Aly for their help and support.

\section{References}

[1] M.A Nielsen and I.L Chuang, Quantum Computation and Quantum Information Cambridge University Press (2010).

[2] P.M. Alsing and G.J. Milburn, Phys. Rev. Lett. 91, 180404 (2003).

[3] A. G. S. Landulfo and G. Matsas, Phys. REv. A 032315 (2009).

[4] S. Khan and M. Khan, J.Phys. A: Math. Theor.,44 355302 (2011).

[5] N. Metwally, arXiv:1201.5941 (2012).

[6] N. Metwally, arXiv:1206.3784 (2012).

[7] Mi-Ra Hwang, D. Park and E. Jung, Phys.Rev.A 83012111 (2001).

[8] B. N. Esfahani and M. Aghaee, Quantum Inf. Process 11529 (2012).

[9] W. Dür, G. Vidal and J. I. Cirac, phys. Rev. A62 062314 (2000).

[10] A. Karlesson and M Bourennane, Phys. Rev. A. 584394 (1998).

[11] S. Bose, V. Vedral and P. Knight, Phys. Rev. A.57 822 (1998).

[12] D. E. Bruschi, J. Louko, E. Martn-Martnez, A. Dragan, and I. Fuentes, Phys. Rev. A 82042332 (2010).

[13] E. M.-Martinez, I. Fuentes, Phys. Rev. A 83, 052306 (2011).

[14] P. M. Alsing, I. F. Schuller, R. B. Mann and T. E. Tessier, Phys. Rev. A 74032326 (2006).

[15] N. Metwally, J. Phys. A :Math. Theor. 44055305 (2011); N. Metwally, J. Opt. Soc. Am. B 29, 389-396 (2012).

[16] K. Yang, L. Huang,W.Yang and F. Song, Int. J. Theor Phys. 48516 (2009).

[17] G. Vidal, R. Werner, Phys. Rev. A.65 032314 (2002). 
[18] Z.-X. Man, Y,-J. Xia and N. Ba An, J. Phys. B:At. Mol. Opt. Phys. 41155501 (2008).

[19] N. Metwall, Int. J. Theor. Phys. 491571 (210); A.-El Allati, N. Metwally and Y. Hassouni,Optics Communications, 284, 519-526 (2011). 